\title{
NECESIDADES PROTEICAS EN INDIVIDUOS FÍSICAMENTE ACTIVOS
}

\author{
Rebeca Hernández \\ Escuela de Educación Física y Deportes \\ Universidad de Costa Rica, San José, Costa Rica \\ E-mail:
}

\begin{abstract}
Resumen
Hernández, R. (2003). Necesidades proteicas en individuos físicamente activos. Revista de Ciencias del Ejercicio y la Salud, 3(1), 63-76. El maximizar la ganancia en masa muscular, ha sido una de las principales preocupaciones de atletas que entrenan la fuerza. Para lograr esto, generalmente consumen elevadas cantidades de proteína dietética en relación a lo establecido por las Recomendaciones Dietéticas Diarias (Recommended Daily Allowances, RDA) norteamericanas $(0.8 \mathrm{~g} / \mathrm{kg} / \mathrm{d})$. Hasta hace pocos años, los requerimientos de proteína en atletas se empezaron a estudiar de manera sistemática. A pesar de que aún se necesita más investigación para determinar recomendaciones precisas, los estudios realizados hasta el momento indican que los requerimientos de proteínas para atletas de resistencia y de fuerza, sí son superiores a los de individuos sedentarios. Se ha sugerido un consumo aproximado de $1.2-1.4 \mathrm{~g} / \mathrm{kg} / \mathrm{d}$ para atletas de resistencia y para atletas que entrenan la fuerza de $1.6-1.7 \mathrm{~g} / \mathrm{kg} / \mathrm{d}$. Existen muchos factores fisiológicos que van a influenciar los requerimientos proteicos. Entre los más importantes se deben destacar la edad, el estado fisiológico, el género y tiempo de entrenamiento (principiantes vs profesionales). Asimismo, se debe considerar que según el tipo de ejercicio, se dan respuestas fisiológicas diferentes, que influyen en la cantidad de proteína requerida. El entrenamiento de fuerza, provocará una aceleración del metabolismo de proteínas, aumentando tanto la síntesis como la degradación. Si se desea favorecer la síntesis, se ha encontrado que una hiperinsulinemia e hiperaminoacidemia simultánea, por medio del consumo de una mezcla de proteína y carbohidrato, durante las primeras tres horas post ejercicio, es la manera más eficiente de lograr las tasas máximas de anabolismo. El ejercicio aeróbico, también aumenta la necesidad de proteína, pero por otros mecanismos. En este caso, lo que se da es un aumento del uso de proteína para energía, en especial Amino Ácidos de Cadena Ramificada (AACR), en especial a intensidades de ejercicio moderadas y altas. Por lo tanto, al realizar ejercicio aeróbico de manera frecuente, lleva a la necesidad de un mayor consumo de proteínas, para así evitar problemas por deficiencia proteica, dada su mayor utilización durante el entrenamiento. Los diferentes estudios apuntan, a que consumir elevadas cantidades de proteína, no representa peligro para la salud de los atletas, siempre que el atleta no tenga historia de enfermedad renal. La mejor manera para asegurar que los requerimientos de proteína se cumplan, es siguiendo una dieta adecuada en energía, así como seleccionar alimentos de alta calidad proteica, como por ejemplo, productos lácteos, huevos, carne, pescado y productos de soya. PALABRAS CLAVES: proteína, Recomendaciones Dietéticas Diarias, atletas, entrenamiento de fuerza, ejercicio, físicamente activo, masa muscular.
\end{abstract}

\section{INTRODUCCIÓN}

Los atletas que entrenan la fuerza, siempre se han preocupado sobre la cantidad de proteína adecuada para maximizar las ganancias en masa muscular. La práctica común de fisicoculturistas y atletas de diferentes disciplinas, ha sido consumir elevadas cantidades de proteína en relación a lo establecido por la Ingesta Dietética de Referencia (Dietary Reference Intake, DRI) norteamericanas $\quad(0.8 \mathrm{~g} / \mathrm{kg} / \mathrm{d}) \quad$ (National Academy of Sciences, 1999).

Esta costumbre, ha sido derivada de manera empírica por los atletas, y durante muchos años los profesionales de la salud y 
deporte, han considerado esta práctica innecesaria, ya que mantenían la posición de que el ejercicio físico no representaba un estímulo suficiente para alterar el metabolismo de proteínas. Debido a esto, la mayoría de las investigaciones se concentraron principalmente sobre el metabolismo de carbohidratos y grasas. Fue hasta la década de los años setenta, que se inició a probar la hipótesis sobre el metabolismo de proteínas y ejercicio, de manera científica y sistemática; y se encontró que la práctica de los atletas de consumir mayores cantidades de proteína podría no estar totalmente errada, e inclusive podría resultar beneficiosa para su rendimiento.

Los principales hallazgos sobre proteína y ejercicio, señalan que para aumentar la masa muscular y fuerza, se necesita una combinación apropiada de los siguientes factores: a. entrenamiento de fuerza; b. hiperinsulinemia fisiológica post ejercicio; c. alta disponibilidad de aminoácidos después de entrenamiento de fuerza (Biolo, Maggi, Williams, Tipton y Wolfe, 1995; Biolo, Tipton, Klein y Wolfe, 1997 ). Inclusive, según lo encontrado por Lemon, Dolny y Yaresheski (1997), el ejercicio aeróbico a intensidades entre 55 $67 \%$ VO2max también puede aumentar los requerimientos de proteína aproximadamente en un $16-25 \%$

Aunque se ha demostrado que la mayoría de las personas mantienen una ingesta proteica superior a la recomendada, es necesario definir de manera concreta los requerimientos. $\mathrm{Al}$ conocer los niveles adecuados, se puede asegurar que todas las personas físicamente activas los estén llenando, y así evitar posibles problemas de salud relacionados a deficiencias, los cuales pueden resultar contraproducentes al rendimiento, en especial en atletas de alto nivel. Además, existen grupos en riesgo de consumir bajas cantidades de proteína, tales como personas con requerimientos superiores debido a su estado fisiológico (crecimiento y embarazo), individuos con bajo consumo energético total y los que consumen proteína de baja calidad (vegetarianos). Para estos últimos, es importante conocer sus requerimientos lo más exacto posible, con el fin de que las intervenciones nutricionales, logren los efectos positivos deseados sobre su salud.

El principal objetivo de esta revisión, es identificar la necesidad de un requerimiento mayor de proteínas en los atletas, analizando los posibles mecanismos fisiológicos que intervienen.

\section{Personas entrenadas vs no entrenadas}

\section{Efecto del entrenamiento}

El grado de entrenamiento de los sujetos, es un factor importante a considerar al interpretar posibles aumentos en los requerimientos proteicos.

Con respecto al entrenamiento aeróbico, se ha notado que la oxidación de amino ácidos para energía es mayor, en los sujetos con más experiencia; probablemente, debido a una mayor actividad de la enzima limitante de la vía oxidativa (oxoácido deshidrogenasa de cadena ramificada), tanto en hígado como en músculo (Lemon, 1997). Esto sugiere que a mayor tiempo de entrenamiento aeróbico, los requerimientos de proteína serán más elevados.

En el entrenamiento de fuerza se ha observado lo contrario. Estudios recientes han demostrado que en individuos entrenados, las tasas de recambio de proteína después del ejercicio de fuerza son menores en comparación con sujetos no entrenados.

Una de las adaptaciones al entrenamiento de fuerza, es la disminución del daño muscular asociado a este ejercicio, por ende la tasa de degradación proteica también disminuye con el tiempo de entrenamiento. (Phillips, Tipton, Ferrando y Wolfe, 1999; Roy, Fowles, Hill y Tarnopolsky. 2002). Este hecho, fue comprobado por Phillips, y cols. (1999), al examinar el efecto del entrenamiento de fuerza en la respuesta de la síntesis proteica fraccional y la degradación de proteína 
fraccional (las mediciones fraccionales se refieren a mediciones de la síntesis y degradación en un músculo específico). Lo encontrado por estos últimos investigadores se puede considerar como altamente confiable, ya que utilizaron para probar su hipótesis, las técnicas de infusión de isótopos estables, y la de biopsia muscular. Éstos, son los métodos más válidos que se han desarrollado para determinar la cinética del metabolismo de las proteínas en respuesta a un estímulo agudo, ya que lo miden directamente.

Esta teoría también se ha respaldado por otros autores, al determinar que las tasas de excreción de nitrógeno ureico y de 3 metilhistidina, de sujetos entrenados son mucho menores que las de sus contrapartes sedentarios (Roy y cols. 2002).

Se debe considerar, que a pesar de que los hallazgos recientes han probado que los sujetos entrenados en fuerza tienen menor degradación proteica en relación a los que están iniciando programas de entrenamiento, los requerimientos proteicos sí serán mayores que los de sujetos sedentarios (Tarnopolsky, Atkinson, MacDougall, Chesley, Phillips y Schwarz, 1992). Si bien, la degradación proteica se minimiza con el tiempo de entrenamiento, el hecho de realizar ejercicio de fuerza siempre va a implicar cierto grado de daño muscular, en relación a cuando no se realiza entrenamiento. Tarnopolsky, y cols (1992), han estimado los requerimientos para individuos que entrenen la fuerza, en aproximadamente $1.79 \mathrm{~g} / \mathrm{kg} / \mathrm{d}$.

\section{Tipo de ejercicio}

\section{a. Ejercicio contra resistencia}

Al realizar entrenamiento contra resistencia, hay una aceleración del metabolismo de proteínas, es decir, tanto la síntesis como la degradación de proteína muscular aumentan (Biolo y cols.1995).

Tarnopolsky y cols. (1992) evaluaron los requerimientos de proteína en atletas que entrenan la fuerza en relación a sus contrapartes sedentarios. Como atletas se tomó un grupo de 7 sujetos que realizaba ejercicios $>4$ días/semana para aumentar la fuerza, y lo había realizado durante al menos dos meses antes del inicio del estudio, 6 sujetos, no entrenados, sanos, actuaron como control. Todos los sujetos participaron en tres experimentos diferentes, cada uno de 13 días, con un período promedio de "blanqueo" de dieta "ad libitum" de 8 días. En cada período, los sujetos fueron asignados aleatoriamente a uno de los tres niveles de ingesta proteica, los cuáles fueron: pobre en proteína $(\mathrm{PP})=0.86$ $\mathrm{g} / \mathrm{kg} / \mathrm{d} ;$ moderada en proteína $(\mathrm{MP})=1.4$ $\mathrm{g} / \mathrm{kg} / \mathrm{d} ;$ y rica en proteína $(\mathrm{RP})=2.4 \mathrm{~g} / \mathrm{kg} / \mathrm{d}$. A partir de los datos de cinética de proteínas, se encontró que la síntesis proteica fue mayor para los atletas en las dietas MP y RP, con respecto a $\mathrm{PP}$, sin diferencias significativas entre dietas. Cuando se consumió la dieta RP, la síntesis no aumentó, en relación a MP, lo que se observó fue aumento en la oxidación de leucina. Esto significa que un consumo elevado de proteína, no es necesario para producir mayor síntesis, ya que el exceso es oxidado como energía y no depositado como proteína muscular. Los individuos sedentarios no aumentaron de manera significativa la síntesis proteica con MP y RP, lo que indica que estos niveles de ingesta representan sobrecarga para personas sedentarias. El balance de nitrógeno sirvió como apoyo de estos hallazgos. Para balance de nitrógeno 0 y un margen de seguridad de +1 DS, los requerimientos estimados fueron: $1.79 \mathrm{~g} / \mathrm{kg} / \mathrm{d}$ para deportistas de fuerza y de $0.89 \mathrm{~g} / \mathrm{kg} / \mathrm{d}$ para sedentarios. Los autores utilizaron un margen de +1 DS y no el +2 DS recomendado por organismos internacionales, por la mayor variación biológica que se obtiene con pequeños grupos de sujetos (13 sujetos participaron en el estudio).

\section{b. Ejercicio aeróbico}

El ejercicio aeróbico regular parece aumentar las necesidades de proteína en aproximadamente $1.2-1.4 \mathrm{~g} / \mathrm{kg} / \mathrm{d}$ (Lemon, y cols. 1997). Aunque los mecansimos exactos 
aún no se han definido de manera concreta, se cree que el incremento en los requerimientos se debe a aumentos en la oxidación de amino ácidos para la producción energía, en especial, los Amino Ácidos de Cadena Ramificada (AACR) (Bowtwell, Leese, Smith, Watt, Nevill, Rooyackers, Wagenmakers y Reenie, 1998; Forslund, ElKhoury, Olsson, Sjodin, Hambreus, y Young, 1999).

Lemon y cols. (1997), demostraron que la actividad aeróbica a intensidad moderada $\left(55 \pm 5.6 \% \mathrm{VO}_{2 \max }\right)$, que es lo que se recomienda para la población en general (US Dept. of Health \& Human Services, 1996), aumenta los requerimientos proteicos diarios sobre el nivel recomendado de 0.8 $\mathrm{g} / \mathrm{kg} / \mathrm{d}$ (RDA). En este estudio, seis sujetos físicamente activos realizaron tres pruebas de una hora de ejercicio en banda sin fin a intensidad baja $\left(42 \quad \pm \quad 3.9 \% \quad \mathrm{VO}_{2 \max }\right)$, moderada $\left(55 \pm 5.6 \% \mathrm{VO}_{2 \max }\right)$ y alta $(67 \pm 4.5$ $\left.\% \mathrm{VO}_{2 \max }\right)$. Para determinar un aumento en el requerimiento, se evalúo el balance de nitrógeno en un período de 3 días, después del estímulo de ejercicio, ya que la finalidad del estudio era determinar el aumento total de los requerimientos y no solamente el efecto agudo del ejercicio. Se debe siempre tener en cuenta, que aunque este método permite medir la respuesta al ejercicio a través del tiempo, no se puede analizar de manera sistemática los cambios en síntesis y degradación proteica.

El ejercicio aeróbico a intensidad moderada y alta, resultó en un aumento en la excreción de nitrógeno ureico de $0-24$ y de 24 - 48 horas post ejercicio. Estos resultados indican que 1 hora de ejercicio moderado aumenta la oxidación de proteínas en aproximadamente 29 - 45 gramos, lo cual representa $16-25 \%$ de las actuales recomendaciones norteamericanas de ingesta diaria de proteína. Esto significa, que los ejercicios que se recomiendan típicamente para mejorar la salud aumentan los requerimientos de proteína en relación a individuos sedentarios o personas que se ejercitan a intensidades menores (Lemon y cols. 1997). En esta investigación, solamente se determinó la respuesta a un solo período de ejercicio en tres días, por lo tanto, si se realiza actividad física con períodos menores de recuperación, es probable que los requerimientos sean mayores a los estimados por Lemon y cols. (1997).

Entre los hallazgos de esta investigación, se debe resaltar también el hecho de que se encontró que las tasas de oxidación de proteínas son mayores a intensidades de ejercicio moderadas $(55 \pm 5.6$ $\left.\% \mathrm{VO}_{2 \max }\right)$ y altas $\left(67 \pm 4.5 \% \mathrm{VO}_{2 \max }\right)$. El mecanismo de esta interacción aún no está claro; se ha propuesto que se debe a una mayor actividad de la enzima oxo - ácido deshidrogenosa de cadena ramificada, como resultado de activación dependiente de la intensidad (Lemon 1998).

\section{Efecto de la edad}

\section{a. Niños}

El American College of Sports Medicine, así como la American Academy of Pediatrics, apoyan la participación de niños en actividades contra resistencia, siempre que los programas sean bien diseñados $y$ supervisados (American College of Sports Medicine, American Dietetic Association y Dietitians of Canada, 2000). También, aumentar la cantidad de niños que participan en actividades que ayudan a mantener la fuerza muscular y la resistencia, es uno de los objetivos de salud pública en los Estados Unidos (U.S. Dept. of Health \& Human Services, 1996).

Investigaciones recientes han

demostrado múltiples beneficios del entrenamiento contra resistencia en la salud infantil. Faigenbaum, Westcott, La Rosa y Long (1999), demostraron que diferentes protocolos de entrenamiento favorecen la fuerza y resistencia muscular tanto en niños como niñas. Otros investigadores, han reportado mejorías en la condición aeróbica, así como una disminución de riesgo de osteoporosis y un aumento en la masa ósea, 
prevención de la obesidad e hipertensión, aumento del colesterol HDL y mejorías en la salud mental (Myers, Strikmiller, Webber, y Berenson, 1994).

Ya que se ha probado que el ejercicio contra resistencia es beneficioso y seguro para los niños, es de suma importancia considerar la utilización de nutrientes durante el ejercicio, debido a la rápida tasa de crecimiento y desarrollo en esta edad. Pikosky, Faigenbaum, Westcott, y Rodríguez (2002), estudiaron el efecto del entrenamiento contra resistencia en 7 niños y 4 niñas entre los 7 y 10 años de edad, sobre los requerimientos proteicos y energéticos. El programa consistió en 6 semanas de entrenamiento contra resistencia dos veces por semana. En el período, la ingesta de proteína de cada niño fue adecuada según el requerimiento $(>1.5 \mathrm{~g} / \mathrm{kg} / \mathrm{d})$.

El balance de nitrógeno promedio aumentó de manera significativa con el entrenamiento, lo que indica un estado de anabolismo, respaldado con el aumento en peso y talla que se observó en los niños. No obstante, este balance positivo, estuvo acompañado de disminuciones significativas en el flujo de proteínas, síntesis proteica y balance neto, lo cual sugiere un posible déficit energético. Este déficit se estimó en $536 \mathrm{kcal} / \mathrm{d}$, a partir de los registros de consumo y el efecto de ejercicio en la tasa de metabolismo basal.

Los autores sugirieron que el cambio en el metabolismo proteico se pudo deber a la "partición de nutrientes", lo cual se refiere, a que los nutrientes se distribuyen de manera diferente en los órganos para poder lograr de manera exitosa la función productiva dominante en el momento, en este caso, el crecimiento. El hecho de que los niños aumentaron peso y talla, masa muscular magra y masa grasa, a pesar de que mostraron un balance energético negativo, presenta evidencia de que probablemente se dio partición de nutrientes, para mantener el crecimiento y desarrollo. Se puede especular que la utilización de proteína para tejidos no relacionados al crecimiento fue disminuida, no obstante, no se puede asegurar, ya que la metodología utilizada no es específica para tejidos determinados.

Si la partición de nutrientes ocurre, significa que se sacrifica el aporte proteico para tejidos no relacionadas al crecimiento, lo cual puede comprometer la función adecuada de ciertos órganos vitales. Esto sugiere que los requerimientos de proteína si se verían aumentados, no obstante, se requiere estudio adicional para determinar la magnitud de esta necesidad .

\section{b. Adulto Mayor}

Al avanzar la edad, hay una pérdida crónica de masa muscular (sarcopenia). No obstante, se ha encontrado que el desempeño muscular puede mejorar en adultos mayores a través de entrenamiento contra resistencia (Yarashesky, Pak - Loduca, Hasten, Obert, Brown y Sinacore, 1999). Debido a esto, es importante determinar si el ejercicio, unido a una suplementación proteica pueden llevar a una mayor ganancia de masa muscular en adultos mayores.

En estudios en los que adultos mayores han realizado entrenamiento contra resistencia, manteniendo la ingesta de proteína y energía según la RDA, sí se han observado aumentos en la masa muscular (Haub, Wells, Tarnopolsky y Campbell, 2002). Esto sugiere que siempre que el consumo de energía y nutrientes sea adecuado, es posible que ocurra hipertrofia en el adulto mayor.

No obstante, se ha encontrado que otros factores pueden influir en la respuesta anabólica del adulto mayor. Por ejemplo, Esmarck, Andersen, Olsen, Richter, Mizuno y Kjaer (2001), sugirieron que el consumo de un suplemento inmediatamente después del ejercicio favorece la ganancia de masa muscular, en relación a cuando se consume dos horas después. Se debe considerar, que en este estudio, los cambios en el metabolismo proteico se infirieron a partir de cambios en la masa muscular. Si se quiere cuantificar directamente la respuesta en el metabolismo 
de proteínas, se deben realizar mediciones directas del recambio proteico, y de la respuesta inmediatamente después del consumo del suplemento, lo cual no se realizó en este caso.

Aún se requiere más investigación para definir de manera concreta las necesidades proteicas del adulto mayor que realiza entrenamiento contra resistencia.

\section{Diferencias según género}

La mayoría de los estudios relacionados con las necesidades proteicas de individuos físicamente activos, se han realizado en hombres, no obstante, estudios recientes apuntan a que la utilización de proteínas en las mujeres que realizan ejercicio aeróbico es menor que en los hombres (Phillips, Atkinson, Tarnopolsky y McDougall, 1993; Carter, Rennie y Tarnopolsky. 2001).

Phillips, y cols. (1993), examinaron lo adecuado de la Ingesta Recomendada de Nutrientes (RNI) Canadiense, vigente en 1993, en atletas de resistencia tanto hombres como mujeres; también cuantificaron el recambio de leucina durante un período prolongado de ejercicio submáximo $(65 \%$ VO2max). A cada sujeto se le dio una dieta isoenergética según su consumo energético habitual, con contenido de proteína según la Ingesta Recomendada de Nutrientes Canadiense $(0.94 \mathrm{~g} / \mathrm{kg} / \mathrm{d}$ para hombres y 0.80 $\mathrm{g} / \mathrm{kg} / \mathrm{d}$ para mujeres) (en Phillips, y cols. 1993).

El balance de nitrógeno demostró que la Ingesta Recomendada de Nutrientes Canadiense para el año 1993, era inadecuada para los que practican de manera crónica ejercicio de resistencia, tanto hombres como mujeres. El hecho de que los sujetos de este estudio fueran entrenados (Hombres $5.2 \pm 2.9$ años, Mujeres $5.6 \pm 2.7$ años), evitó que se pueda detectar un efecto del inicio del entrenamiento.

Los resultados del estudio de Phillips y cols. (1993), demostraron diferencias importantes según género. En los hombres, se encontró una mayor oxidación de leucina (expresada en $\mathrm{kg}$ de peso corporal, así como también en $\mathrm{kg}$ de masa muscular magra), lo que puede ocasionar que tengan requerimientos mayores que las mujeres atletas. En las mujeres, el cociente de intercambio respiratorio (RER), reflejó una mayor oxidación de lípidos. Se cree que esta mayor oxidación de lípidos, ayuda a conservar el glucógeno muscular, reduciendo el uso de proteína para gluconeogénesis.

Por otro lado, Carter y cols. (2001), evaluaron la utilización de macronutrientes durante el ejercicio realizando un estudio longitudinal, lo que permitió comparar los efectos del inicio del entrenamiento. Estos investigadores encontraron, que tanto antes y después de un período de entrenamiento en resistencia, las mujeres utilizan menor cantidad de carbohidratos y aminoácidos y mayor cantidad de grasa, en relación a los hombres.

En el entrenamiento de fuerza y contra resistencia, no se han realizado investigaciones que demuestren diferencias sexuales en la respuesta anabólica.

\section{Balance energético}

Cuando la dieta no contiene adecuadas cantidades de energía, la síntesis proteica se reduce, los amino ácidos se oxidan para generar la energía faltante y, consecuentemente aumentan los requerimientos de proteína (Torún, Menchú y Elías, 1996). En los atletas que restringen la ingesta de alimentos, esto puede resultar aún más contraproducente, ya que como se explicó, los requerimientos ya están aumentados por el ejercicio, tanto en atletas de fuerza como de resistencia.

También, parece que existen diferencias sexuales al ajustar la ingesta energética según el gasto. Se ha observado que las mujeres atletas, no llegan a adaptar su ingesta a un gasto energético aumentado (Fig 1.) (Lemon, 2000). 
Fig. 1. Diferencias sexuales en ingesta energética de individuos físicamente activos

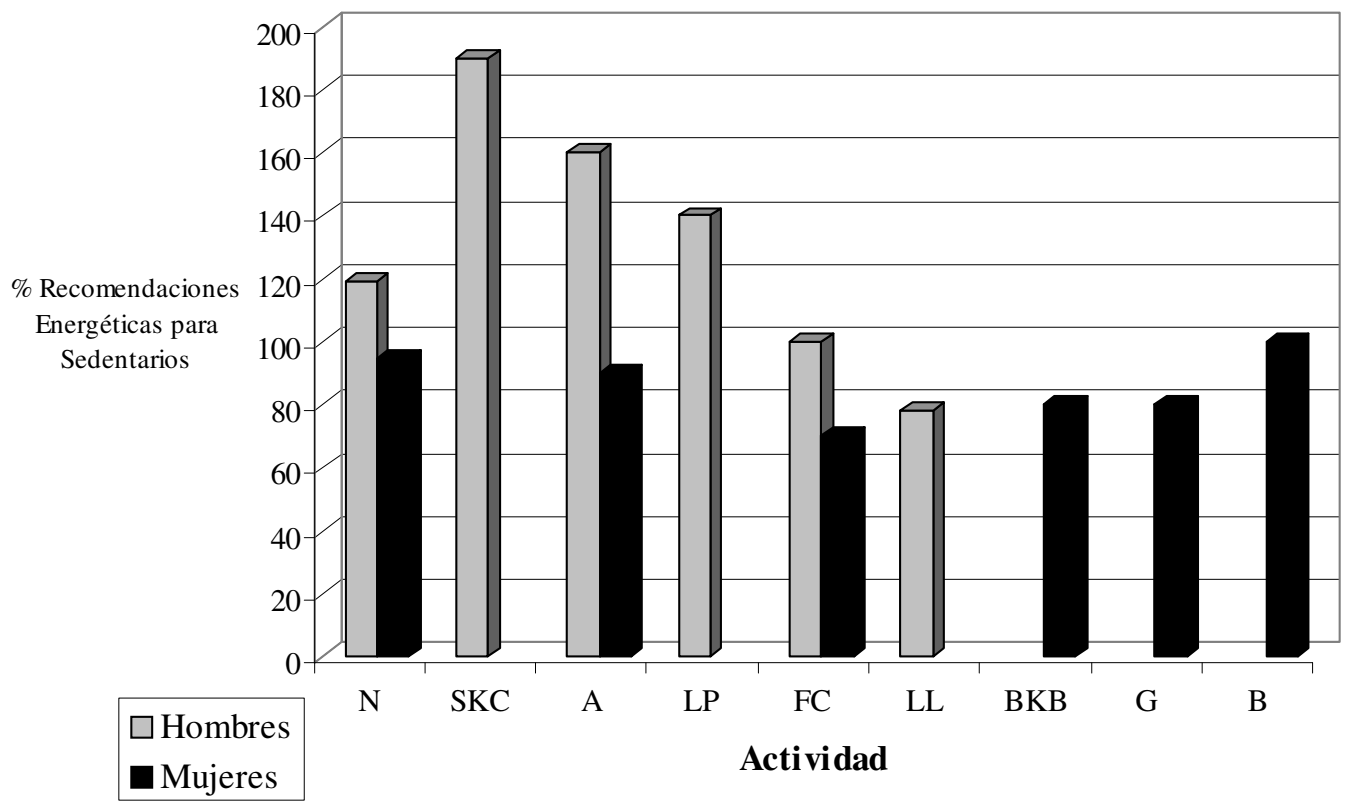

Nota: N: natación; SKC: ski cross - country; A: atletismo; LP: levantamiento de pesas; FC: fisicoculturismo; LL: lucha libre; BKB: basketball; G: gimnasia; B: ballet. Los hombres, en su mayoría, aumentan su ingesta energética de manera apropiada según el gasto que su actividad implica. Las mujeres no logran llenar su gasto energético (Adaptado de Lemon, 2000).

Se ha demostrado que un aumento en proteína dietética reduce las pérdidas de nitrógeno cuando se mantiene una dieta baja en energía. También, se ha reportado que un mayor aporte de proteína dietética, específicamente AACR, ayuda a mantener la síntesis proteica en condiciones catabólicas (Layman, Boileau, Erickson, Painter, Shiue, Sather y Christou, 2003). Por lo tanto, en grupos de atletas en riesgo de consumir dietas hipocalóricas, el consumo de proteína sobre las recomendaciones actuales para personas sedentarias, puede ser beneficioso para mantener la masa muscular. Layman, y cols. (2003), demostraron que para personas con sobrepeso una dieta hipocalórica, moderada en proteína $(1.6 \mathrm{~g} / \mathrm{kg} / \mathrm{d})$ produce una disminución similar de peso que una dieta hipocalórica normal en proteína $(0.8 \mathrm{~g} / \mathrm{kg} / \mathrm{d})$, no obstante la dieta moderada en proteína es más eficiente para mejorar la composición corporal. Los cambios en la relación pérdida de grasa/pérdida de masa magra demostraron que el grupo que mantuvo mayor ingesta proteica, mejoró la utilización de las grasas, mientras mantuvo constante la masa magra. El mecanismo de este efecto se desconoce; no obstante, se encontró en el estudio que al sustituir proteína por carbohidrato en una dieta hipocalórica se mantienen los niveles plasmáticos de T3 (tri - yodotironina) y T4 (tiroxina), además de favorecer una respuesta insulínica menor a los comidas. Estas diferencias en el perfil hormonal son consistentes con una mayor tasa de lipólisis, por lo que la dieta alta en proteína es más eficiente en conservar proteína muscular al favorecer la utilización de grasa para energía. En este estudio no se reportó la cantidad de actividad física que realizaron los sujetos, por 
lo que es probable que la cantidad de proteína requerida por los atletas para mantener masa magra sea mayor.

\section{Contenido de carbohidrato}

Se ha encontrado que la disponibilidad de carbohidrato (CHO), está relacionado de manera inversa a la tasa de catabolismo proteico.

Forslund, y cols. (1999) evaluaron el efecto de consumir una dieta normoproteica $(1 \mathrm{~g} / \mathrm{kg} / \mathrm{d})$ o hiperproteica $(2.5 \mathrm{~g} / \mathrm{kg} / \mathrm{d})$ en combinación con ejercicio físico (2 períodos de 90 minutos a intensidad $45-50 \%$, VO2 max) sobre la producción de energía y la tasa de utilización de macronutrientes en un período de 24 horas. Los investigadores encontraron que la disponibilidad de macronutrientes tiene un importante efecto en la tasa de utilización de éstos para la producción de energía. La ingesta mayor de carbohidrato en el grupo de dieta normoproteica, favoreció una mayor oxidación de carbohidrato; de la misma manera, el grupo de dieta alta en proteína, presentó una mayor contribución de proteína y menor contribución de carbohidrato a la producción de energía, tanto en valores absolutos como relativos. Estos resultados sugieren, que para las personas que practican ejercicio aeróbico a intensidades moderadas, es de suma importancia mantener una dieta con una relación $\mathrm{CHO}$ /proteína balanceada, ya que un bajo consumo de carbohidrato, disminuye la cantidad de proteína disponible para síntesis, debido a que se estimula su uso para producción de energía. Cabe resaltar que es difícil extender estos resultados a todo atleta, ya que la utilización de macronutrientes durante el ejercicio se ve afectada por muchos otros factores, entre estos, intensidad del ejercicio, estado nutricional y el tipo de entrenamiento que han tenido los sujetos. En este caso se evaluó ejercicio aeróbico a intensidades de baja a moderada (45 - 50\% VO2 max) en hombres físicamente activos, no atletas de élite.

\section{Tiempo de ingesta}

Uno de los factores más relevantes con respecto a favorecer la hipertrofia muscular es el tiempo de ingesta de macronutrientes. Se ha demostrado que a pesar de que el entrenamiento contra resistencia por sí solo favorece la síntesis proteica, si el individuo permanece en estado post absortivo después de este estímulo, la degradación excede la síntesis proteica, resultando más bien en pérdida de tejido muscular (Biolo, y cols. 1997).

Las primeras investigaciones sobre el tema, utilizaron infusiones intravenosas de aminoácidos después del entrenamiento contra resistencia para favorecer el anabolismo. Este método fue efectivo en provocar ganancia neta de masa muscular, aunque poco práctica para los atletas interesados. Tipton, Ferrando, Phillips, Doyle y Wolfe (1999), demostraron que ingerir un suplemento oral inmediatamente después del entrenamiento provoca aumentos similares en la síntesis proteica. Estos investigadores también hicieron énfasis, en que en el suplemento que se administre, los amino ácidos no esenciales no son necesarios para estimular la síntesis proteica; una dosis de solamente amino ácidos esenciales es suficiente. Se determinó, que aproximadamente $6 \mathrm{~g}$ totales de aminoácidos esenciales son suficientes para maximizar la tasa de síntesis proteica.

La mayoría de las investigaciones recientes coinciden con lo encontrado por Tipton, y cols. (1999). En el estudio realizado por Ramussen, Tipton, Millar, Wolf y Wolfe (2000), un suplemento de $6 \mathrm{~g}$ de amino ácidos esenciales en combinación con $35 \mathrm{~g}$ de sacarosa consumido una hora después o tres horas después de realizar ejercicio contra resistencia, resultó en un estímulo anabólico similar.

El mecanismo exacto a través del cual se favorece el anabolismo, aún no se ha determinado de manera concreta; no obstante, sí se han descrito varios factores que parecen 
favorecer la respuesta anabólica al entrenamiento de fuerza.

Por ejemplo, el ambiente hormonal que se crea después de la ingesta de nutrientes ha sido uno de los temas más estudiados. Se ha probado que la ingesta de un suplemento oral que contenga amino ácidos esenciales y carbohidratos, provoca el mismo aumento en las concentraciones plasmáticas de insulina que una bebida que contenga solamente carbohidrato (Roy, y cols. 2002). Inclusive, se ha visto que la respuesta insulínica puede ser mayor al combinar proteína y carbohidrato. van Loon, Kruijshoop, Verhagen, Saris y Wagenmakers (2000), encontraron que después de un protocolo de depleción de reservas de glucógeno, la respuesta insulínica es mayor cuando se consume una mezcla de hidrolizado de proteína, leucina y fenilalanina libres y carbohidrato, en relación a cuando se consume carbohidrato solamente. Además, encontraron que esta respuesta fue dependiente de la dosis, a mayor concentración de hidrolizado de proteína, leucina y fenilalanina libres, mayor fue la respuesta insulínica.

Estudios recientes concuerdan, en que la principal acción de la insulina no radica en aumentar la síntesis proteica, por el contrario, actúa disminuyendo la degradación de masa muscular que ocurre como consecuencia del ejercicio de fuerza (Ramsussen, y cols. 2000; Tipton, Ramussen, Millar, Wolf, Owens Stovall, Petrini y Wolfe, 2001).

Otro factor que ha sido estudiado, es el que se dé hiperaminoacidemia en conjunto con hiperinsulinemia. La disponibilidad de aminoácidos esenciales es un factor limitante de la síntesis proteica. Se ha demostrado, que para que el estímulo anabólico del entrenamiento de fuerza sea efectivo, es vital la ingesta de amino ácidos esenciales post ejercicio. Una combinación de entrenamiento de fuerza, hiperinsulinemia, e hiperaminoacidemia, parece tener un efecto aditivo; porque las mayores tasas de síntesis proteica se han reportado cuando estos factores ocurren de manera simultánea (Ramussen, y cols. 2000)

Tipton, y cols. (2001) sugieren que la ingesta de un suplemento pre ejercicio, produce un mayor aumento en la síntesis proteica, y por lo tanto mayor ganancia de masa muscular. Estos investigadores propusieron que esta respuesta puede acontecer debido al aumento del flujo sanguíneo hacia el músculo esquelético que ocurre durante el ejercicio. Este aumento, favorece el transporte de aminoácidos hacia el compartimiento intracelular; por lo tanto, hay mayor disponibilidad en el momento en que la síntesis está aumentada.

Kraemer, Volek, Bush, Putukian y Sebastianelli (1998), describieron la respuesta hormonal anabólica (insulina, hormona del crecimiento, IGF - 1 (Insulin like Growth Factor - 1)) aguda, a la ingesta de un suplemento de carbohidrato - proteína (rico en AACR), después de un período de entrenamiento de fuerza. En la investigación participaron 9 hombres entrenados en fuerza, cada sujeto actuó como su propio control. Durante 7 días de tratamiento, los sujetos consumieron el suplemento o una cantidad equivalente de placebo. La composición del suplemento fue la siguiente: $33 \%$ proteína (albúmina y caseína predigeridas), $67 \%$ carbohidrato (polímeros de glucosa, glucosa, fructuosa cristalina, y xilitol). El placebo se diseñó con el fin de que el sabor y la apariencia fueran iguales al suplemento, aportando un mínimo de carbohidrato, proteína y calorías. Este placebo contenía xilitol, celulosa microcristalina, polvo de cocoa, extracto de crema deshidratada, goma guar, aspartame y sabor natural de chocolate. Los últimos tres días de cada tratamiento los sujetos realizaron ejercicio de fuerza. El suplemento se consumió dos horas antes del ejercicio e inmediatamente después del ejercicio.

Cuando se consumió el suplemento, los análisis indicaron que sí hubo diferentes respuestas hormonales en relación a cuando no se consumió el suplemento. Concretamente, aumentaron las 
concentraciones de glucosa, insulina, hormona del crecimiento e IGF -1 , y disminuyeron, las concentraciones de lactato. Estas respuestas indican que se favorece el anabolismo sobre el catabolismo, aumentándose la síntesis de glucógeno y proteína en el período de recuperación. Se debe tener presente que estos análisis se realizaron en atletas, por lo que la respuesta puede ser diferente en individuos no entrenados. Una mayor respuesta anabólica se comprueba por el hecho de que el promedio de masa corporal fue mayor durante la condición de suplementación en relación a la condición placebo. Se puede considerar que esta diferencia se debió principalmente a masa magra, ya que no hubo diferencias en pliegues cutáneos entre condiciones.

\section{Calidad proteica}

Campbell, Barton, Cyr-Campbell, Davey, Beard, Parise. y Evans (1999), evaluaron si el consumo de carne en una dieta omnívora podía influir en los cambios inducidos por un período de 12 semanas de entrenamiento de fuerza en composición corporal y tamaño de músculo esquelético en hombres adultos mayores, en comparación con una dieta lactoovovegetariana.

Con el entrenamiento de fuerza, la densidad corporal total, y masa muscular corporal total aumentaron en el grupo de dieta mixta pero disminuyeron en el grupo de dieta lactoovovegetariana. Por el contrario, el porcentaje promedio de grasa corporal aumentó en el grupo lactoovovegetariano y disminuyó en el grupo que mantuvo su dieta omnívora habitual. Los investigadores concluyeron, que el consumo de una dieta que contiene carne contribuye a obtener ganancias mayores en masa muscular total al combinarse con entrenamiento de fuerza, en comparación a cuando durante el entrenamiento de fuerza se consume una dieta lactoovovegetariana.

Cabe resaltar, que contrario a lo observado en estudios posteriores, el grupo de dieta lactoovovegetariana tuvo pérdidas de peso. A pesar de que se mantuvieron registros de consumo para controlar las ingestas totales de macronutrientes, los hombres que ingirieron la dieta lactoovovegetariana tuvieron ingesta total de proteína menor, lo que puede contribuir a un balance neto negativo, y al mayor catabolismo.

Una posible explicación al favorecimiento del anabolismo por consumir proteína animal, ha sido dada por Volek, Kraemer, Bush, Incledon y Boetes (1997), quienes encontraron que los nutrientes de la dieta influencian las concentraciones de testosterona, hormona que favorece el anabolismo. Las concentraciones de testosterona se relacionan de manera negativa y significativa con la relación de ácidos grasos polinsaturados - ácidos grasos saturados. Al disminuir el consumo de fuentes de proteína animal, al adoptarse una dieta vegetariana, la proporción de ácidos grasos polinsaturados a saturados aumenta, por lo que es probable que ocurra una disminución de la testosterona, haciendo que el efecto anabólico sea menor. No obstante, esto no se puede comprobar en el estudio de Campbell, y cols. (1999), debido a que no se midieron las concentraciones plasmáticas de testosterona.

Contrario a estos hallazgos, Haub, y cols. (2002), no encontraron diferencias en los aumentos de masa muscular entre individuos que consumieron una dieta mixta en comparación con los que consumieron una dieta lactoovovegetariana. En este estudio se tuvo mejor control de la ingesta energética y proteica de los participantes, para ambos grupos fue adecuada según los requerimientos. Por lo tanto, estos datos sugieren que aumentos en fuerza muscular y tamaño muscular no son influenciados por la fuente de proteína consumida por hombres adultos mayores, siempre que la ingesta proteica sea adecuada.

Aún se requiere más investigación para determinar si realmente existe diferencia en la respuesta anabólica según el origen de la proteína. 


\section{Riesgos de dietas altas en proteína}

Una de las principales interrogantes que puede emerger al recomendarse una ingesta superior a las RDA actuales, es si es seguro este consumo, con respecto a posibles consecuencias renales. Un elevado contenido de proteínas en la alimentación aumenta el trabajo de los riñones debido a la carga adicional de nitrógeno que debe ser eliminada, pero esto no parece ser un problema para las personas sanas. Poortmans y Dellalieux (2000), estudiaron fisicoculturistas y otros atletas entrenados, con ingestas de proteína elevadas y moderadamente altas en relación a las actuales RDA. Los atletas completaron un registro de consumo de siete días, y se les realizaron análisis de sangre y recolecciones de orina con el fin de determinar las posibles consecuencias renales del alto consumo de proteína. A pesar de las elevadas concentraciones plasmáticas de ácido úrico y calcio, en el grupo de fisicoculturistas, las tasas de aclaramiento de creatinina, urea y albúmina, se encontraron dentro de rangos normales. Cabe destacar que no se encontraron correlaciones entre la ingesta de proteína y la tasa de depuración de creatinina, la tasa de excreción de albúmina y la tasa de excreción de calcio. Por lo tanto, a partir de este estudio, se puede concluir que ingestas de proteína de hasta $2.8 \mathrm{~g} / \mathrm{kg} / \mathrm{d}$ no afectan la función renal en atletas entrenados.

Otro riesgo que se puede asociar al aumentar el consumo de proteína, es un mayor consumo de fuentes de origen animal. Las dietas altas en proteína animal se han asociado a mayor riesgo de enfermedad cardiovascular, ya que aportan altas cantidades de colesterol y grasa saturada, no obstante, este hecho parece no ser problemático. En el estudio realizado por Layman, y cols. (2003), mujeres con sobrepeso fueron asignadas a una dieta hipocalórica moderada en proteína $(1.6 \mathrm{~g} / \mathrm{kg} / \mathrm{d})$ o una dieta normoproteica $(0.8 \mathrm{~g} / \mathrm{kg} / \mathrm{d})$. El colesterol total y el colesterol LDL disminuyeron en porcentaje similar en ambos grupos, no obstante, la concentración de triglicéridos plasmáticos solamente disminuyó de manera significativa en el grupo de dieta moderada en proteína. En el estudio se evaluó directamente la relación entre proteína y carbohidrato sustituyendo alimentos fuente de proteína animal (carnes rojas, productos lácteos, huevos) por alimentos altos en carbohidratos (panes, arroz, pasta y cereales), manteniendo constante la ingesta de energía, fibra y grasa. El grupo de dieta moderada en proteína tuvo una ingesta de colesterol total de más del doble que el grupo de dieta normoproteica. El hecho de que ambos grupos disminuyeran en igual porcentaje el colesterol total y el LDL, sugiere que otros factores son más importantes en determinar los niveles de colesterol plasmático que el colesterol dietético. Layman, y cols. (2003) también demostraron que la dieta alta en proteína estimuló la lipólisis, favoreciéndose así la utilización de grasa para energía.

\section{CONCLUSIONES}

A pesar de que aún se necesita más investigación para determinar recomendaciones precisas, los estudios realizados hasta el momento sugieren que los requerimientos de proteínas para atletas de resistencia son de aproximadamente 1.2-1.4 $\mathrm{g} / \mathrm{kg} / \mathrm{d}$ y para los atletas que entrenan la fuerza de $1.6-1.7 \mathrm{~g} / \mathrm{kg} / \mathrm{d}$.

Son muchos factores que intervienen fisiológicamente en las necesidades proteicas de los atletas. En referencia a los atletas entrenados en fuerza, los hallazgos más recientes indican que el entrenamiento de fuerza produce una aceleración del metabolismo de proteínas, aumentando tanto la síntesis como la degradación. Si se desea favorecer la síntesis, se ha encontrado que la hiperinsulinemia y la hiperaminoacidemia simultánea, como resultado de la ingesta de una mezcla de al menos $6 \mathrm{~g}$ de amino ácidos esenciales y carbohidrato durante las primeras tres horas post ejercicio, es la manera más eficiente de lograr las tasas máximas de 
anabolismo. Es importante considerar que en individuos entrenados, las tasas de recambio de proteína después del ejercicio de fuerza son menores en relación a sujetos no entrenados, por lo que aún se necesitan más estudios sobre los efectos del entrenamiento para determinar requerimientos según el individuo sea novato o más experimentado.

Respecto a diferentes requerimientos según edad, se ha estudiado los dos extremos del continuo, niños y adultos mayores. En los niños que realizan entrenamiento contra resistencia, se ha observado que el crecimiento y el aumento en masa muscular no se ven comprometidos, aún cuando durante los períodos de entrenamiento se mantenga un balance energético negativo. Esto sugiere, que el organismo "distribuye" los nutrientes de manera tal, que se mantienen las funciones más importantes del momento, en este caso el crecimiento, y se sacrifica el aporte de nutrientes a otros tejidos. Si esto ocurre, significa que los niños que realicen entrenamiento contra resistencia deben consumir mayor cantidad de proteína que sus contrapartes sedentarios, ya que aunque el crecimiento se mantiene, es probable que algunos tejidos de órganos vitales no estén recibiendo los nutrientes suficientes para un funcionamiento adecuado. Se requiere más investigación en niños, para definir de manera precisa, cuáles son las cantidades adecuadas de consumo.

En el adulto mayor que realiza entrenamiento contra resistencia, la necesidad de un mayor consumo de proteína aún no se ha dilucidado, ya que los estudios han tenido hallazgos contradictorios. En la edad avanzada, es probable que las respuestas hormonales ocurran de manera diferente en comparación con adultos jóvenes, por lo que es necesario que se estudie más este tema.

En cuanto a diferencias según género, en el caso del ejercicio aeróbico, se han documentado desigualdades claras en la utilización de macronutrientes durante el ejercicio. Se ha encontrado que los hombres utilizan mayor proporción de proteína como energía, lo que puede ocasionar que tengan requerimientos mayores que las mujeres. La magnitud de esta diferencia, aún no se ha definido. En el entrenamiento de fuerza y contra resistencia, no se han realizado investigaciones que demuestren diferencias en la respuesta anabólica según sexo.

La composición de la dieta en general, también es de suma importancia en relación a la utilización de proteína. Primero que todo, si se mantiene un balance energético negativo, y si la ingesta de carbohidrato es inadecuada, la mayoría de la proteína se utiliza para producir energía faltante, reduciéndose la cantidad disponible para síntesis.

La mejor manera para asegurar que los requerimientos de proteína sean llenados, es el consumir una dieta adecuada en energía, así como seleccionar alimentos de alta calidad proteica, por ejemplo, productos lácteos, huevos, carne, pescado y productos de soya.

\section{BIBLIOGRAFÍA}

American College of Sports Medicine, American Dietetic Association and Dietetians of Canada. (2000) Joint position statement: Nutrition and athletic performance. Medicine and Science in Sports and Exercise, 32(12), 2130 - 2145.

Biolo, G., Maggi, S.P., Williams, B.D., Tipton, K.D. \& Wolfe, R.R. (1995). Increased rates of muscle protein turnover and amino acid transport after resistance exercise in humans. American Journal of Physiology Endocrinology and Metabolism 268(3), E514 - 520

Biolo, G., Tipton, K.D., Klein, S. \& Wolfe, R.R. (1997). An abundant supply of amino acids enhances the metabolic effect of exercise on muscle protein. American Journal of Physiology Endocrinology and Metabolism 273, E122 - 129

Bowtwell, J.L., Leese, G.P., Smith, K., Watt, P.W., Nevill, A., Rooyackers, O., Wagenmakers, A.J.M. \& Reenie, M.J. ( 1998 ). Modulation of whole body protein metabolism, during and after exercise, by variation of dietary protein. Journal of Applied Physiology, 85(5), 1744 - 1752.

Campbell, W.W., Barton Jr., M.L., Cyr-Campbell, D., Davey, S.L., Beard, J.L, Parise, G. \& Evans, W.J. (1999). Effects of an omnivorous diet compared with a lactoovovegetarian diet on resistance - training - induced changes in body and skeletal muscle in older men. American Journal of Clinical Nutrition, 70, 1032 - 1039 
Carter, S.L., Rennie, C. \& Tarnopolsky, M.A. (2001) Substrate utilization during endurance excercise in men and women after endurance training. American Journal of Endocrinology Metabolism, 280, E898 - E907.

Esmarck, B.., Andersen, J.L., Olsen, S., Richter, E.A., Mizuno, M. \& Kjaer, M. (2001). Timing of postexercise intake is important for muscle hypertrophy with resistance training in elderly humans. Journal of Physiology, 535(1), 301 311.

Faigenbaum, A.D., Westcott, W.L, La Rosa - Loud, R. \& Long, C. (1999). The effects of different resistance training protocols on muscular strength and endurance development in children. Pediatrics, 104(1). 1 - 7-

Forslund, A.H., El - Khoury, A.E., Olsson, R.M., Sjodin, A.M., Hambraeus, L. \& Young, V.R. (1999). Effect of protein intake and physical activity on 24 - hour pattern and rate of macronutrient utilization. American Journal of Physiology, 276, E964 - E976.

Haub, M.D, Wells, A.M., Tarnopolsky, M.A. \& Campbell, W.W. (2002). Effect of protein source on resistive - training induced changes in body composition and muscle size in older men. American Journal of Clinical Nutrition, 76(3), $511-517$.

Kraemer, W.J., Volek, J.S., Bush, J.A., Putukian, \& Sebastianelli, W.J. (1998). Hormonal responses to consecutive days of heavy - resistance exercise with or without nutritional supplementation. Journal of Applied Physiology 85 (4), 1544 - 1555

Layman, D.K., Boileau, R.A, Erickson, D.J. Shiue. H. Sather,C. and Christou, D. \& Painter, J.E. (2003).A reduced ratio of dietary carbohydrate to protein improves body composition and blood lipid profiles during weight loss in adult women. Journal of Nutrition, 133, 411 - 417

Lemon, P.W.R. (1998). Effects of exercise on dietary protein requirements. International Journal of Sports Nutrition Exercise and Metabolism 8, $426-444$

Lemon, P.W.R. ( 2000 ). Beyond the zone : protein needs of active individuals. Journal of the American College of Nutrition, 19(5), 513S $521 \mathrm{~S}$.

Lemon, P.W.R. Dolny, D.G., \& Yarasheski, K.E. (1997) Moderate physical activity can increase dietary protein needs. Canadian Journal of Applied Physiology, 22(5), 494 - 503.

Myers, L, Strikmiller, P.K., Webber, L.S. \& Berenson, G.S. (1994). Physical and sedentary activity in school childeren grades 5 - 8: the Bogalusa Herat Study. Medicine and Science in Sports and Exercise, 28, 510 - 514.
National Academy of Sciences. (1999). Dietary Reference Intakes. Washington, DC: National Academy Press.

Phillips, S.M., Atkinson, S.A., Tarnopolsky \& MacDougall, J.D. (1993). Gender differences in leucine kinetics and nitrogen balance in endurance athletes. Journal of Applied Physiology, 75(5), 2134 - 2141

Phillips, S.M., Tipton, K.D., Ferrando, A.A. \& Wolfe, R.R. (1999). Resistance training reduces the acute exercise induced increased in muscle protein turnover. American Journal of Physiology $276 \quad$ (Endocrinology and Metabolism 39), E118 - 124

Pikosky, M., Faigenbaum, A., Westcott, W. \& Rodriguez, N. (2002). Effects of resistance training on protein utilization in healthy children. Medicine and Science in Sports and Exercise, 34(5), 820 - 827.

Poortmans \& Dellalieux. (2000). Do regular high protein diets have potential health risks on kidney function in athletes? International Journal of Sports Nutrition Exercise and Metabolism, 10(1), 28 - 38

Ramussen, B.B., Tipton, K.D., Miller, S.L., Wolf, S.E. and Wolfe, R.R. (2000). An oral essential amino acid - carbohydrate supplement enhaces muscle protein anabolism after resistance exercise. Journal of Applied Physiology 88, 386 - 392

Roy, B.D., Fowles, J., Hill, R. \& Tarnopolsky, M.A. (2002). Macronutrient intake and whole body protein metabolism following resistance exercise. Medicine and Science in Sports and Exercise, 32(8), 1412 - 1419

Tarnopolsky, M.A., Atkinson, S.A., MacDougall, J.D., Chesley, A., Phillips, S. \& Shwarz, H.P. (1992). Evaluation of protein requirements for trained strength athletes. Journal of Applied Physiology 73, $1986-1995$

Tipton, K.D., Ferrando, A.A., Phillips, S.M., Doyle, D. \& Wolfe, R.R.(1999). Postexercise net protein synthesis in human muscle from orally administered amino acids. American Journal of Physiology 276 (Endocrinology and Metabolism 39), E628 - 634

Tipton, K.D., Ramussen, B.B., Millar, S.L., Wolf, S.E., Owens - Stovall, S.K., Petrini, B.E. \& Wolfe, R.R. (2001). American Journal of Physiology Endocrinology and Metabolism 281, E197 - 206

Torún, B., Menchú, M.T. \& Elías, L.G. ( 1996 ). Recomendaciones Dietéticas Diarias del INCAP. (edición XLV ANIVERSARIO). Ciudad Guatemala: Publicación INCAP.

U.S. Dept. of Health \& Human Services. (1996). Physical activity and health: A report form the Surgeon General. U.S. Dept of Health and Human Services, Centers for Disease Control and Prevention, National Center for Chronic Disease Prevention and Health Promotion, Atlanta, GA. 
van Loon, L.J.C., Kruijshoop, M., Verhagen, H., Saris, W.H.M. \& Wagenmakers, A.J.M. (2000). Ingestion of protein hydrolysate and amino acid - carbohydrate mixtures increase postexercise plasma insulin responses in men. Jorunal of Nutrition 130, 2508 - 2513.

Volek, J.S., Kraemer, W.J., Bush, J.A., Incledon, T. \& Boetes, M. (1997). Testosterone and cortisol in relationship to dietary nutrients and resistance exercise. Journal of Applied Physiology, 82(1), $49-54$.

Yarashesky, K.E., Pak - Loduca, J., Hasten, D.L., Obert, Brown, K.A. \& Sinacore, D.R.. (1999). Resistance exercise training increases mixed muscle protein synthesis rate in frail men and women $\geq 76$ years old. American Journal of Physiology Endocrinology and Metabolism, 277, E118 - E125. 